\title{
Bench Test of a Residual Gas Ionization Profile Monitor (RGIPM)*
}

\author{
W. C. Sellyey, J. D. Gilpatrick, LANL, Los Alamos NM, USA \\ Ralph Senior, General Atomics, San Diego CA, USA
}

\section{Abstract}

An RGIPM has been designed ${ }^{1}$, constructed and bench tested to verify that all components are functioning properly and that the desired resolution of about $50 \mu \mathrm{m}$ rms can be achieved. This paper will describe major considerations that went into the bench test and some results.

\section{INTRODUCTION}

The components of the system will be described in varying detail emphasising items and concepts relevant to the bench test. The components intended for a beam line are a magnet for producing a uniform magnetic (B) field that is perpendicular to the beam direction, a vacuum box that contains electrodes for generating a uniform electric (E) field parallel to the B field and a detector to measure the electron distribution. If a beam passes through the vacuum box it ionizes residual gas. The $\mathrm{E}$ and $\mathrm{B}$ fields guide the resulting electrons to the detector located well outside the beam at the surface of the vacuum box. For the bench test, an ultraviolet light illuminates $100 \mu \mathrm{m}$ wire, thus simulates the beam. In both cases, the electron distribution is observed using a scintillation detector that moves perpendicular to the wire or beam and perpendicular to the fields.

\section{MAGNET}

Figure 1 shows a top view of the magnet. It was designed using OPERA-3D (Vector Fields). The dimensions of the two iron cores are $91 \mathrm{~cm}$ by $64 \mathrm{~cm}$ by 8 $\mathrm{cm}$. There is $6 \mathrm{~cm}$ between the two cores. The maximum gap between the pole tips is $24.66 \mathrm{~cm}$ and they have a small parabolic curvature so the gaps at the top and bottom are $24.3 \mathrm{~cm}$.

The magnet was designed to produce a field of $.08 \mathrm{~T}$ and was operated at $.12 \mathrm{~T}$ with fans cooling the coils. The magnet was not mapped and thus only OPERA-3D field information is available. For this discussion the beam direction is the $\mathrm{Z}$ direction, the direction parallel to the pole tips (without curvature) and perpendicular to the beam is the $\mathrm{X}$ direction. The ideal $\mathrm{B}$ and $\mathrm{E}$ field direction is the $\mathrm{Y}$ direction.

If there is a $\mathrm{B}_{Z}\left(\mathrm{~B}_{\mathrm{Z}}\right.$ is the $\mathrm{Z}$ component of $\mathrm{B}$ etc.) it will cause the electrons to drift in the $X$ direction ${ }^{2}$. If $\left(\mathrm{dB}_{\mathrm{Z}} / \mathrm{dX}\right) / \mathrm{B}_{\mathrm{Y}}$ is not zero it will contribute to the spread of the electron distribution at the detector. The largest value for this is $9.2 * 10^{-5} / \mathrm{mm}$ in the region where the electrons

* Work supported by the US DOE, Defense Programs and by the US DOE, Nuclear Energy. move. It can be shown that $(\mathrm{Et} / \mathrm{B})\left(\mathrm{dB}_{\mathrm{Z}} / \mathrm{dX}\right) / \mathrm{B}$ is the fractional change in the size of an electron distribution at the detector due to $\mathrm{dB}_{\mathrm{Z}} / \mathrm{dX}$. E and $\mathrm{B}$ represent the field magnitudes and $t$ is the transit time from the wire to the detector. Typically $\mathrm{t}=4.5 \mathrm{~ns}, \mathrm{E}=64.5 \mathrm{kV} / \mathrm{m}$ and $\mathrm{B}=0.12 \mathrm{~T}$. This gives a fractional change of $0.22 \mu \mathrm{m} / \mathrm{mm}$.

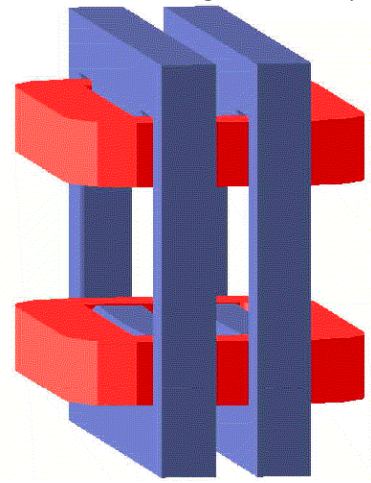

Figure 2. Top View of Magnet; Beam moves across the paper and $\mathrm{B}$ is up or down.

\section{ELECTROSTATICS}

Figure 2 shows one half of a cross section of the vacuum box inside of which are the electrodes for generating a nearly uniform electric field in the $\mathrm{Y}$ direction. The beam direction is perpendicular to the page. An $8 \mathrm{~mm}$ thick Al plate is the negative electrode. The vacuum box is the grounded positive electrode. A set of $100 \mu \mathrm{m}$ wires (diameter greatly exaggerated) $1 \mathrm{~cm}$ from the $\mathrm{Al}$ plate, separated by $3 \mathrm{~cm}$ were designed to be suppression grids. In the bench test, they are used as a source of electrons with narrow spatial distribution to simulate a beam. At the lower right side of the vacuum box in figure 2, there is an arrow identifying the location of a $100 \mu \mathrm{W}$ Krypton light and collimator. It shines a $5^{\circ}$ wide beam on the wires near the center of the box and these photons cause the wires to emit electrons.

A detector assembly moves in a $1.8 \mathrm{~cm}$ wide by $10 \mathrm{~cm}$ long slot parallel to the $\mathrm{Z}$ direction at $\mathrm{X}=0$ and $\mathrm{Y}=157.5$ $\mathrm{mm}$. A 125 or $400 \mu \mathrm{m}$ hole defined a limiting aperture. The electrons that pass through this hole hit a $0.5 \mathrm{~mm}$ thick, $2 \mathrm{X} 2 \mathrm{~mm}$ square scintillator. A $500 \mu \mathrm{m}$ gold coated quartz optical fiber caries some of the resulting photons to a photomultiplier (PM) tube outside the vacuum.

The equipotential surfaces in figure 2 were obtained using the 2D electrostatic code PC-OPERA (Vector Fields). For this simulation the plate was set to $-10 \mathrm{~V}$ and the grid wires at $-9.264 \mathrm{~V}$. The plate and vacuum box are approximately rectangular, so the fields obtained this way cannot be used for precise calculations. However, they should be adequate for error estimation. $\left(\mathrm{dE}_{\mathrm{Z}} / \mathrm{dX}\right) / \mathrm{E}_{\mathrm{Y}}$ is 
the field characteristic that determines how seriously the resolution is effected by $\mathrm{E}$ field variations from the ideal. This is a 2-D solution so there is no $E_{Z}$. To get a rough estimate $\left(\mathrm{dE}_{\mathrm{X}} / \mathrm{dX}\right) / \mathrm{E}_{\mathrm{Y}}$ will be used. The largest value for this ratio is $1.5^{*} 10^{-4} / \mathrm{mm}$ in the region where the electrons move. The fractional change in the size of an electron distribution at the detector is given by $(\mathrm{t} / \mathrm{B})\left(\mathrm{dE}_{\mathrm{Z}} / \mathrm{dX}\right)$ and it evaluates to $0.36 \mu \mathrm{m} / \mathrm{mm}$ for the same values of $\mathrm{t}, \mathrm{B}$ and $\mathrm{E}$ used in section 2 .

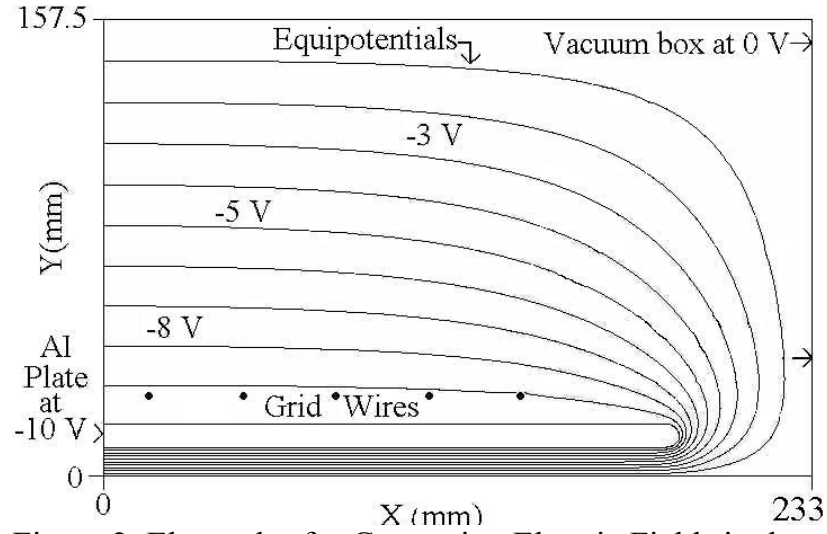

Figure 2. Electrodes for Generating Electric Fields in the $\mathrm{Y}$ direction and also showing Equipotentials.

It will be demonstrated below that the fields near the wire acting on the electrons can be divided into two types. One is a dipole like field resulting from the approximately uniform field that would exist if the wires were not present. This field will oppose the electron emission on the side of the wire facing the negative plate, and will accelerate electrons leaving the other side. The second type of field is a monopole and can be represented by a line charge near the center of the wire. This field will add to the dipole field on one side of the wire, and subtract on the other side. By controlling the relative strength of the monopole to the dipole fields, the parts of the wire that emit electrons can, in principle, be controlled. This is done by supplying a stable grid to plate voltage ratio.

Figure 3 is a schematic of a voltage divider that was used to supply this stable high voltage ratio. A common supply was used in combination with precision resistors. A potentiometer is at high voltage between two corona rings. Its value is adjusted manually by means of an insulated shaft. The adjustment is needed for reasons described above. It is also needed because the voltage cannot be readily calculated for two reasons. One is the resistor precision limits the calculation to about $0.2 \%$ of the power supply voltage. The second is that in order to determine what the ratio should be, very precise electric field distributions in the vacuum box are needed and this is difficult to do. An empirical approach was chosen here and an expression for the potentials to be used is derived below.

The solution to the problem of infinitely long charged conducting cylinder of charge $\lambda$ per unit length above an infinite conducting grounded plate is well known ${ }^{3}$. Let the plate be at $Y=0$, in the $X-Z$ plane. Let the center of the charged cylinder be at $\mathrm{Y}=\mathrm{d}, \mathrm{X}=0$ and let the radius of the cylinder be a. The fields can be described by two line (image) charges, one at $\mathrm{Y}_{0}=\mathrm{d}-\mathrm{a}^{2} / 2 \mathrm{~d}, \mathrm{X}=0$ with charge $\lambda$ and a second at $-Y_{0}, X=0$ with charge $-\lambda$. In the situation that is of interest here, $\mathrm{d}=10 \mathrm{~mm}$, and $\mathrm{a}=50 \mu \mathrm{m}$.

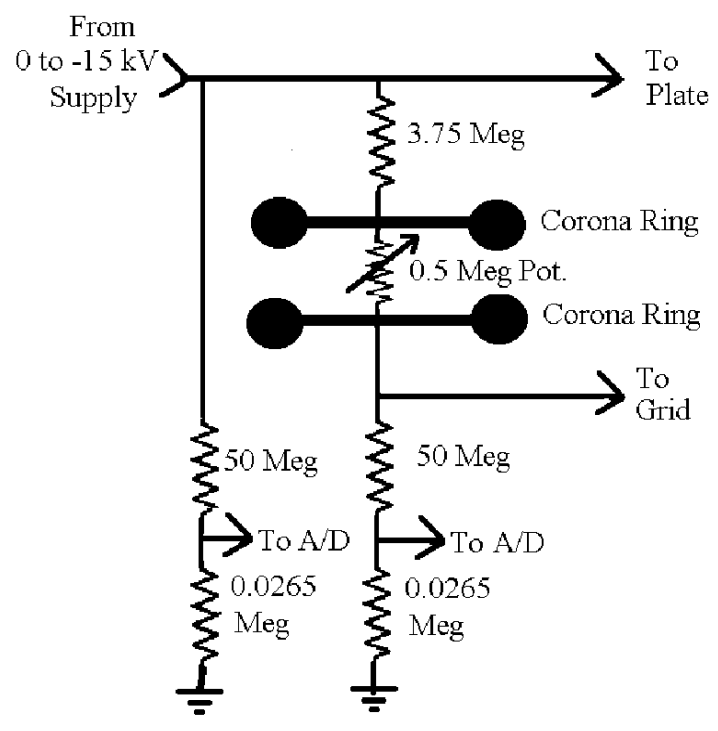

Figure 3. High Voltage Divider

Adding a second infinite conducting surface at $Y_{1}=139$ $\mathrm{mm}$ parallel to the one at $\mathrm{Y}=0$, will require an infinite set of image charges for an exact solution. It can be shown that this infinite series of image charges contributes less than $2 \%$ to the electric field magnitude within $10 \mathrm{~mm}$ of the wire.

The field resulting when an infinite conducting cylinder of radius ' $a$ ' is placed in a uniform infinite extent electric field of magnitude $\mathrm{E}_{0}$ is also well known ${ }^{3}$. The new field is the original field plus a dipole field whose magnitude is $\mathrm{E}_{0}(\mathrm{a} / \mathrm{r})^{2}$, where $\mathrm{r}$ is the distance from the cylinder center. Let the cylinder be the $100 \mu \mathrm{m}$ wire located between two infinite plates described above, and suppose the plates are charged so the field in the absence of the wire is $\mathrm{E}_{0}$. It can be shown that the field with the plates in place differs from the infinite extent field by a factor of less than $10^{-5} \mathrm{E}_{0}$.

As can be seen in figure 2, there is a total of 10 grid wires. They all generate dipole and monopole fields. For the central wires, the field contribution of the dipole components from the other wires is $10^{-5} \mathrm{E}_{0}$. The monopole component adds about $10 \%$ to the potential on the central wires compared to what this potential would be with only one wire.

In the actual system partly described by figure 2, the plates are finite. $\mathrm{E}_{0}$ at the central wires calculated in this $2 \mathrm{D}$ geometry is $3.1 \%$ greater than for infinite plates and the voltage at the wire center changes by $3.2 \%$. The real system is three-dimensional and has many surface irregularities. However, within $1 \mathrm{~cm}$ of a central grid wires in the field direction away from the Al plate, and 1 
$\mathrm{mm}$ in the direction perpendicular to the field direction, the dipole plus monopole model should be valid especially if, as is done here, $\mathrm{E}_{0}$ and the grid potential are left as parameters to be determined from profile data. The radius of curvature of a $1 \mathrm{keV}$ electron in a $0.1 \mathrm{~T}$ magnetic field is $750 \mu \mathrm{m}$ and this is why only $1 \mathrm{~mm}$ is needed in the $\mathrm{X}$ direction. Figure 4 shows that motion perpendicular to $\mathrm{B}$ is $<300 \mu \mathrm{m}$.

\section{DATA AND ANALYSIS}

Photoelectrons are guided to the detector by the parallel electric and magnetic fields. The detector is scanned across this distribution and this results in a count rate distribution that is a convolution of the detector aperture and the electron distribution. The data acquisition system is described elsewhere ${ }^{4}$. Figure 4 shows three background corrected scans taken with a plate voltage of $6 \mathrm{kV}$ and three different $\mathrm{B}$ fields. The detector collimator diameter was $125 \mu \mathrm{m}$.

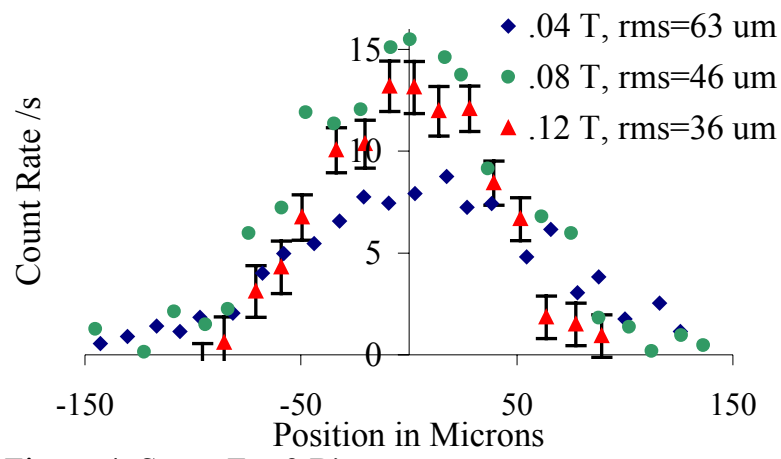

Figure 4. Scans For 3 B's

Software was written to describe the process that the photoelectrons go through from the time they absorb the energy of a photon, to when they arrive at the scintillator ${ }^{4}$. The first part of the program traces the electrons in the gold of the $100 \mu \mathrm{m}$ wire and is Monte Carlo based. Once an electron leaves the metal, its motion is determined by the uniform magnetic field, and an initially rapidly changing electric field described in the section 3 . The path of the electrons is traced to the detector using $\mathrm{F}=\mathrm{ma}$ in 0.1 ps steps.

Figure 5 shows example of the distributions that results at the detector for various grid voltages. The uniform $\mathrm{E}$ field for this was $64.5 \mathrm{~V} / \mathrm{m}$. The voltage shown on the legend is the grid voltage. This is defined as the voltage needed to generate the monopole term. The measured profiles are analysed as convolutions of these distributions with the shape of the detector collimator. Examples of this have been shown elsewhere ${ }^{4}$.

Figure 6 shows the fit to the background corrected measured areas for scans taken at a $0.08 \mathrm{~T}$ magnetic field, $6 \mathrm{kV}$ plate voltage while varying the grid potentiometer with a $400 \mu \mathrm{m}$ detector collimator. The calculated points were generated using the software just described. When the measured data were obtained, only the potentiometer setting was known. The change in grid voltage with potentiometer setting is calculated from the resistor values of figure 3 as .0518 volts per division for a plate voltage of $6000 \mathrm{~V}$. This is used to convert the potentiometer readings to relative voltage. These relative voltages are displaced and the measured count rates are scaled until the measured data closely coincides with the calculated curve.

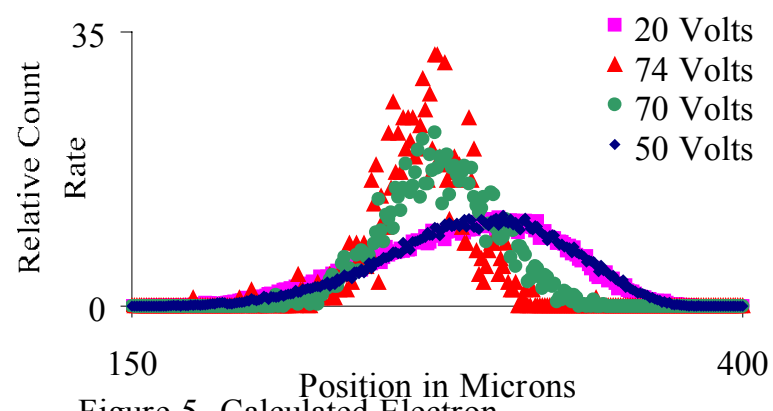

Figure 5. Calculated Electron

Distributions

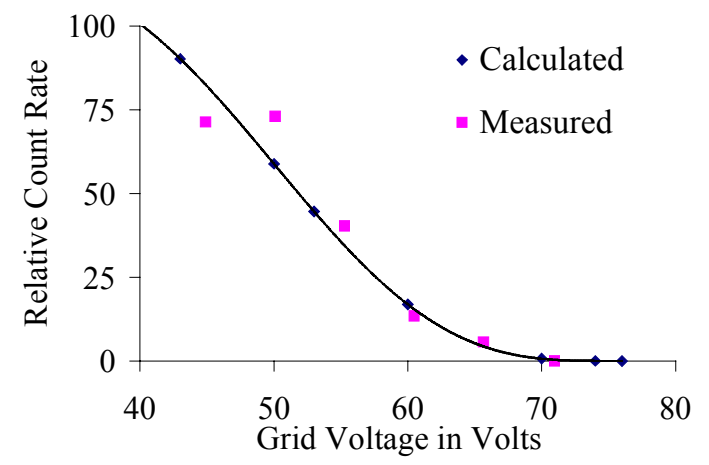

Figure 6. Count Rate v.s. Grid Voltage

\section{PROBLEMS AND CONCLUSIONS}

One of the data points taken to generate figure 6 was not used. Its grid voltage value is about $48 \mathrm{~V}$. Its relative count rate would be 90 and this would put it well above the curve in figure 6 . This data point was taken one day before the rest of the points. The anomalous result was probably a consequence of inadequate tensioning of the wires. Vibration and temperature changes could easily have resulted in wire motion of $350 \mu \mathrm{m}$ to move the point off the curve. Thus spring tensioning is recommended.

The mechanical profile scanning system has many difficulties and needs improvement or replacement with some other type of readout.

\section{REFFERENCES}

[1] W. C. Sellyey, J. D. Gilpatrick, "A Compact Residual Gas Ionization Profile Monitor System", Proceedings of the 1999 PAC, 2152.

[2] J. D. Jackson, "Classical Electrodynamics", John Wiley \& Sons, 1963.

[3] E. M. Pugh, E. W. Pugh, "Principles of Electricity and Magnetism", Addison-Wesley, 1970

[4] W. C. Sellyey, J. D. Gilpatrick, R. Senior, "Bench Test of a Residual Gas Ionization Profile Monitor (RGIPM)", DIPAC 2001, 5/13/01, Grenoble, France. 